\title{
BIVARIATE MONOTONE APPROXIMATION
}

\author{
GEORGE A. ANASTASSIOU
}

(Communicated by J. Marshall Ash)

\begin{abstract}
Let $f$ be a two variable continuously differentiable real-valued function of certain order on $[0,1]^{2}$ and let $L$ be a linear differential operator involving mixed partial derivatives and suppose that $L(f) \geq 0$. Then there exists a sequence of two-dimensional polynomials $Q_{m, n}(x, y)$ with $L\left(Q_{m, n}\right) \geq$ 0 , so that $f$ is approximated simultaneously and uniformly by $Q_{m, n}$. This approximation is accomplished quantitatively by the use of a suitable twodimensional first modulus of continuity.
\end{abstract}

\section{INTRODUCTION}

An essential topic of approximation theory is of monotone approximation, initiated by O. Shisha in 1965 (see [13]). There the problem was: given a positive integer $r$, approximate with rates a given function whose $r$ th derivative is $\geq 0$ by polynomials having the same property. This initial problem was generalized by G. A. Anastassiou and O. Shisha in 1985 (see [1]) by replacing the $r$ th derivative with a linear differential operator of order $r$. The rate of the related uniform convergence was given through the first modulus of continuity.

During the last twenty-five years there has been extensive research on monotone polynomial approximation, in particular, improving Shisha's initial result e.g. J. A. Roulier [12]. Especially G. G. Lorentz and K. Zeller [8], G. G. Lorentz [7], and then R. DeVore [3] have obtained Jackson type estimates on the rate of uniform approximation of monotone functions by monotone polynomials. Furthermore E. Passow, L. Raymon, and J. A. Roulier [10, 11] have studied deeply the comonotone polynomial approximation of comonotone functions and D. J. Newman [9] was able to produce a Jackson type estimate related to comonotone approximation.

More recently R. DeVore and X. Yu [4] have given a constructive proof of Timan-Teljackovski type pointwise estimates for monotone polynomial approximation involving the second modulus of smoothness $\omega_{2}$. Also D. Leviatan [5] presented pointwise estimates involving $\omega_{2}$ and providing convex polynomial

Received by the editors June 9, 1989 and, in revised form, September 14, 1990.

1980 Mathematics Subject Classification (1985 Revision). Primary 41A10, 41A28, 41A29; Secondary $41 \mathrm{~A} 25$. 
approximation, as well as simultaneous monotone and convex polynomial approximation. In addition, using a suitable Peetre functional, D. Leviatan [6] obtained estimates with respect to $\omega_{2}$ of the Jackson type on the rate of monotone polynomial approximation. Then he applied these results to get estimates on the degree of comonotone polynomial approximation.

In this paper we deal with the following general two-dimensional problem (see Theorem 2): Let $f$ be a two variable continuously differentiable real-valued function of given order and let $L$ be a linear differential operator involving mixed partial derivatives and suppose that $L(f) \geq 0$. Then find a sequence of bivariate polynomials $Q_{m, n}(x, y)$ with the property $L\left(Q_{m, n}\right) \geq 0$ so that $f$ is approximated simultaneously in $Q_{m, n}$ in the uniform norm. This approximation is given with rates through inequalities involving the bivariate first modulus of continuity in the Stancu sense (see [14]). We prove our result with the help of a bivariate simultaneous approximation theorem by I. Badea and C. Badea (see [2]).

We would like to mention

Definition (D. D. Stancu [14]). Let $f \in C\left([0,1]^{2}\right),[0,1]^{2}=[0,1] \times[0,1]$, where $\left(x_{1}, y_{1}\right),\left(x_{2}, y_{2}\right) \in[0,1]^{2}$ and $\delta_{1}, \delta_{2} \geq 0$. The first modulus of continuity of $f$ is defined as follows:

$$
\omega\left(f, \delta_{1}, \delta_{2}\right)=\sup _{\substack{\left|x_{1}-x_{2}\right| \leq \delta_{1} \\\left|y_{1}-y_{2}\right| \leq \delta_{2}}}\left|f\left(x_{1}, y_{1}\right)-f\left(x_{2}, y_{2}\right)\right|
$$

Definition 2. Let $f$ be a real-valued function defined on $[0,1]^{2}$ and let $m, n$ be two positive integers. Let $B_{m, n}$ be the Bernstein (polynomial) operator of order $(m, n)$ given by

$B_{m, n}(f ; x, y)=\sum_{i=0}^{m} \sum_{j=0}^{n} f\left(\frac{i}{m}, \frac{j}{n}\right) \cdot\left(\begin{array}{c}m \\ i\end{array}\right) \cdot\left(\begin{array}{c}n \\ j\end{array}\right) \cdot x^{i} \cdot(1-x)^{m-i} \cdot y^{j} \cdot(1-y)^{n-j}$.

For integers $r, s \geq 0$, we denote by $f^{(r, s)}$ the differential operator of order $(r, s)$, given by

$$
f^{(r, s)}(x, y)=\partial^{r+s} f(x, y) / \partial x^{r} \partial y^{s} .
$$

To prove Theorem 2 we need the following simultaneous approximation result:

Theorem 1 (I. Badea, C. Badea [2]). It holds that

$$
\begin{aligned}
\left\|f^{(k, l)}-\left(B_{m, n} f\right)^{(k, l)}\right\|_{\infty} \leq & t(k, l) \cdot \omega\left(f^{(k, l)} ; \frac{1}{\sqrt{m-k}}, \frac{1}{\sqrt{n-l}}\right) \\
& +\max \left\{\frac{k(k-1)}{m}, \frac{l(l-1)}{n}\right\} \cdot\left\|f^{(k, l)}\right\|_{\infty},
\end{aligned}
$$

where $m>k \geq 0, n>l \geq 0$ are integers, $f$ is a real-valued function on $[0,1]^{2}$ such that $f^{(k, l)}$ is continuous, and $t$ is a positive real-valued function on $\mathbf{N}^{2}$, $\mathbf{N}=\{0,1,2, \ldots\}$. Here $\|\cdot\|_{\infty}$ is the supremum norm. 
Next comes our result

Theorem 2. Let $h_{1}, h_{2}, v_{1}, v_{2}, r, p$ be integers, $0 \leq h_{1} \leq v_{1} \leq r, 0 \leq h_{2} \leq$ $v_{2} \leq p$ and let $f \in C^{r, p}\left([0,1]^{2}\right)$. Let $\alpha_{i j}(x, y), i=h_{1}, h_{1}+1, \ldots, v_{1}$; $j=h_{2}, h_{2}+1, \ldots, v_{2}$ be real-valued functions, defined and bounded in $[0,1]^{2}$ and assume $\alpha_{h_{1} h_{2}}$ is either $\geq \alpha>0$ or $\leq \beta<0$ throughout $[0,1]^{2}$. Consider the operator

$$
L=\sum_{i=h_{1}}^{v_{1}} \sum_{j=h_{2}}^{v_{2}} \alpha_{i j}(x, y) \partial^{i+j} / \partial x^{i} \partial y^{j}
$$

and suppose that throughout $[0,1]^{2}$,

$$
L(f) \geq 0 \text {. }
$$

Then for integers $m, n$ with $m>r, n>p$, there exists a polynomial $Q_{m, n}(x, y)$ of degree $(m, n)$ such that $L\left(Q_{m, n}(x, y)\right) \geq 0$ throughout $[0,1]^{2}$ and

$$
\left\|f^{(k, l)}-Q_{m, n}^{(k, l)}\right\|_{\infty} \leq \frac{P_{m, n}(L, f)}{\left(h_{1}-k\right) !\left(h_{2}-l\right) !}+M_{m, n}^{k, l}(f),
$$

all $(0,0) \leq(k, l) \leq\left(h_{1}, h_{2}\right)$. Furthermore we get

$$
\left\|f^{(k, l)}-Q_{m, n}^{(k, l)}\right\|_{\infty} \leq M_{m, n}^{k, l}(f),
$$

for all $\left(h_{1}+1, h_{2}+1\right) \leq(k, l) \leq(r, p)$. Also (4) is true whenever $0 \leq k \leq h_{1}$, $h_{2}+1 \leq l \leq p$ or $h_{1}+1 \leq k \leq r, 0 \leq l \leq h_{2}$. Here

$$
\begin{aligned}
M_{m, n}^{k, l} \equiv M_{m, n}^{k, l}(f) \equiv & t(k, l) \cdot \omega\left(f^{(k, l)} ; \frac{1}{\sqrt{m-k}}, \frac{1}{\sqrt{n-l}}\right) \\
& +\max \left\{\frac{k(k-1)}{m}, \frac{l(l-1)}{n}\right\} \cdot\left\|f^{(k, l)}\right\|_{\infty}
\end{aligned}
$$

and

$$
P_{m, n} \equiv P_{m, n}(L, f) \equiv \sum_{i=h_{1}}^{v_{1}} \sum_{j=h_{2}}^{v_{2}} l_{i j} \cdot M_{m, n}^{i, j},
$$

where $t$ is a positive real-valued function on $\mathbf{N}^{2}$ and

$$
l_{i j} \equiv \sup _{(x, y) \in[0,1]^{2}}\left|\alpha_{h_{1} h_{2}}^{-1}(x, y) \cdot \alpha_{i j}(x, y)\right|<\infty .
$$

Proof. Let $m, n$ be integers such that $m>r, n>p$.

Case (i). Assume that throughout $[0,1]^{2}, \alpha_{h_{1} h_{2}} \geq \alpha>0$. From Theorem 1 we have

$$
\left\|\left(f+P_{m, n} \cdot \frac{x^{h_{1}}}{h_{1} !} \cdot \frac{y^{h_{2}}}{h_{2} !}\right)^{(k, l)}-\left(Q_{m, n}(x, y)\right)^{(k, l)}\right\|_{\infty} \leq M_{m, n}^{k, l},
$$


all $0 \leq u \leq r, 0 \leq l \leq p$, where

$$
Q_{m, n}(x, y) \equiv B_{m, n}(f ; x, y)+P_{m, n} \cdot \frac{x^{h_{1}}}{h_{1} !} \cdot \frac{y^{h_{2}}}{h_{2} !} .
$$

When $(0,0) \leq(k, l) \leq\left(h_{1}, h_{2}\right),(5)$ becomes

$$
\left\|f^{(k, l)}(x, y)+P_{m, n} \frac{x^{h_{1}-k}}{\left(h_{1}-k\right) !} \cdot \frac{y^{h_{2}-l}}{\left(h_{2}-l\right) !}-Q_{m, n}^{(k, l)}(x, y)\right\|_{\infty} \leq M_{m, n}^{k, l} .
$$

Hence, by the triangle inequality property of $\|\cdot\|_{\infty}$ and $(x, y) \in[0,1]^{2}$ we have the validity of inequality (3). Furthermore, if $(x, y) \in[0,1]^{2}$, then

$$
\begin{aligned}
\alpha_{h_{1} h_{2}}^{-1}(x, y) \cdot L\left(Q_{m, n}(x, y)\right)= & \alpha_{h_{1} h_{2}}^{-1}(x, y) \cdot L(f(x, y)) \\
& +P_{m, n}+\sum_{i=h_{1}}^{v_{1}} \sum_{j=h_{2}}^{v_{2}} \alpha_{h_{1} h_{2}}^{-1}(x, y) \cdot \alpha_{i j}(x, y) \\
& \cdot\left[Q_{m, n}(x, y)-f(x, y)-P_{m, n} \frac{x^{h_{1}}}{h_{1} !} \cdot \frac{y^{h_{2}}}{h_{2} !}\right]^{(i, j)} \\
\geq & P_{m, n}-\sum_{i=h_{1}}^{v_{1}} \sum_{j=h_{2}}^{v_{2}} l_{i j} \cdot M_{m, n}^{i, j}=0,
\end{aligned}
$$

the last is true by inequality (5). Therefore $L\left(Q_{m, n}(x, y)\right) \geq 0$.

Case (ii). Assume that throughout $[0,1]^{2}, \alpha_{h_{1} h_{2}} \leq \beta<0$. From Theorem 1 we have

$$
\left\|\left(f-P_{m, n} \frac{x^{h_{1}}}{h_{1} !} \frac{y^{h_{2}}}{h_{2} !}\right)^{(k, l)}-\left(Q_{m, n}(x, y)\right)^{(k, l)}\right\|_{\infty} \leq M_{m, n}^{k, l},
$$

all $0 \leq u \leq r, 0 \leq l \leq p$, where

$$
Q_{m, n}(x, y) \equiv B_{m, n}(f ; x, y)-P_{m, n} \frac{x^{h_{1}}}{h_{1} !} \cdot \frac{y^{h_{2}}}{h_{2} !} .
$$

When $(0,0) \leq(k, l) \leq\left(h_{1}, h_{2}\right),(6)$ becomes

$$
\left\|f^{(k, l)}(x, y)-P_{m, n} \frac{x^{h_{1}-k}}{\left(h_{1}-k\right) !} \cdot \frac{y^{h_{2}-l}}{\left(h_{2}-l\right) !}-Q_{m, n}^{(k, l)}(x, y)\right\|_{\infty} \leq M_{m, n}^{k, l} .
$$

Hence, by the triangle inequality property of $\|\cdot\|_{\infty}$ and $(x, y) \in[0,1]^{2}$ we 
have the validity of inequality (3). Furthermore, if $(x, y) \in[0,1]^{2}$, then

$$
\begin{aligned}
\alpha_{h_{1} h_{2}}^{-1}(x, y) \cdot L\left(Q_{m, n}(x, y)\right)= & \alpha_{h_{1} h_{2}}^{-1}(x, y) \cdot L(f(x, y)) \\
& -P_{m, n}+\sum_{i=h_{1}}^{v_{1}} \sum_{j=h_{2}}^{v_{2}} \alpha_{h_{1} h_{2}}^{-1}(x, y) \cdot \alpha_{i j}(x, y) \\
& \cdot\left[Q_{m, n}(x, y)-f(x, y)+P_{m, n} \frac{x^{h_{1}}}{h_{1} !} \cdot \frac{y^{k_{2}}}{h_{2} !}\right]^{(i, j)} \\
\leq & -P_{m, n}+\sum_{i=h_{1}}^{v_{1}} \sum_{j=h_{2}}^{v_{2}} l_{i j} \cdot M_{m, n}^{i, j}=0,
\end{aligned}
$$

the last is true by inequality (6). Therefore, again $L\left(Q_{m, n}(x, y)\right) \geq 0$.

In the cases of either $\left(h_{1}+1, h_{2}+1\right) \leq(k, l) \leq(r, p)$ or $\left(0 \leq k \leq h_{1}\right.$, $\left.h_{2}+1 \leq l \leq p\right)$ or $\left(h_{1}+1 \leq k \leq r, 0 \leq l \leq h_{2}\right)$, we have

$$
\left(f \pm P_{m, n} \frac{x^{h_{1}}}{h_{1} !} \cdot \frac{y^{h_{2}}}{h_{2} !}\right)^{(k, l)}=f^{(k, l)} \text {. }
$$

Thus inequalities (5) and (6) imply inequality (4). The theorem is now established.

Remark. To the best of our knowledge our result is the first one in the multivariate polynomial monotone approximation. Therefore some questions are raised.

(i) Could we remove the assumption that $\alpha_{h_{1} h_{2}}$ is either $\geq \alpha>0$ or $\leq \beta<0$ throughout $[0,1]^{2}$ ?

(ii) Could we obtain a better rate of uniform or pointwise convergence, and which one is the optimal. What about the $L_{p}$ case?

(iii) Could we involve in our inequalities a higher order modulus of smoothness?

\section{REFERENCES}

1. G. A. Anastassiou and O. Shisha, Monotone approximation with linear differential operators, J. Approx. Theory 44 (1985), 391-393.

2. I. Badea and C. Badea, On the order of simultaneous approximation of bivariate functions by Bernstein operators, Anal. Numér. Théor. Approx. 16 (1987), 11-17.

3. R. A. DeVore, Monotone approximation by polynomials, SIAM J. Math. Anal. 8 (1977), 906-921.

4. R. A. DeVore and X. M. Yu, Pointwise estimates for monotone polynomial approximation, Constr. Approx. 1 (1985), 323-331.

5. D. Leviatan, Pointwise estimates for convex polynomial approximation, Proc. Amer. Math. Soc. 98 (1986), 471-474.

6. _ Monotone and comonotone polynomial approximation revisited, J. Approx. Theory $\mathbf{5 3}$ (1988), 1-16. 
7. G. G. Lorentz, Monotone approximation, Inequalities III (O. Shisha, ed.), Academic Press, New York, 1972, pp. 201-215.

8. G. G. Lorentz and K. Zeller, Degree of approximation by monotone polynomials. I, J. Approx. Theory 1 (1968), 501-504.

9. D. J. Newman, Efficient co-monotone approximation, J. Approx. Theory 25 (1979), 189192.

10. E. Passow and L. Raymon, Monotone and comonotone approximation, Proc. Amer. Math. Soc. 42 (1974), 340-349.

11. E. Passow, L. Raymon, and J. A. Roulier, Comonotone polynomial approximation, J. Approx. Theory 11 (1974), 221-224.

12. J. A. Roulier, Monotone approximation of certain classes of functions, J. Approx. Theory 1 (1968), 319-324.

13. O. Shisha, Monotone approximation, Pacific J. Math. 15 (1965), 667-671.

14. D. D. Stancu, Studii Si Cercetari Stiintifice, XI (2) (1960), 221-233.

Department of Mathematical Sciences, Memphis State University, Memphis, TENNESSEE 38152 